\title{
The Influence of Laser Irradiation Time on Fe-Si-C System Structure
}

\author{
A. Amulevicius, A. Daugvila, R. Davidonis and K. Mazeika \\ Institute of Physics, Savanoriu 231, 2653 Vilnius, Lithuania
}

(Received April 14, 2003; revised version October 20, 2003)

\begin{abstract}
The influence of different laser irradiation times on the structure of grey cast iron was studied. Mössbauer spectroscopy and X-ray diffraction were used to characterise the changes caused by laser irradiation. The composition of martensite and austenite as well as the creation of carbide phases during quenching were studied. Modelling of temperature during laser irradiation allowed differences in the influence of different laser irradiation types and times to be explained.
\end{abstract}

PACS numbers: 61.18.Fs, 61.10.-i, 61.80.Ba

\section{Introduction}

Laser irradiation was used to modify the surface of steel [1-4], create alloys, synthesise new compounds, and partially crystallise amorphic material [5-11]. It can also be used to modify an alloy by inserting material deposited on its surface. In this case, it is important to a void evaporating the material while melting the surface layer [12].

Many studies have been devoted to the laser processing of steels including studies of impurity distributions under different laser treatment conditions [6]. The essence of modification is the saturation of steel with carbon and substitutional impurities and the creation of a disperse structure.

The modification of the properties of grey cast iron ( $\mathrm{Fe}-\mathrm{Si}-\mathrm{C})$ using laser and ultrasound treatment was studied in [13]. The transformation (austenitisation) of grey cast iron by conventional tempering was studied using Mössbauer spectroscopy in [14]. Grey cast iron, in which carbon is in the form of graphite and the percentage of carbon is sufficiently large, is an interesting and favourable material for studying the influence of laser irradiation. During laser irradiation, 
the carbon in the grey cast iron does not evaporate and successfully mixes with the iron [15], unlike when iron is alloyed with carbon from the surface layer [5].

The structure of the alloys depends on a number of homogeneous and heterogeneous processes occurring while quenching the melt. Quenching the melt enables the high-temperature structure, which is oversaturated with impurities and consists of non-equilibrium phases, to be fixed.

The peculiarities of the influence of different types of laser irradiation (continuous scanned and pulsed laser irradiation) on these materials have still not been adequately explored. Our experiments are intended to study the composition of samples and the distribution of impurities in the iron lattice, which are the consequences of the interaction of laser irradiation with the sample surface. The task of modelling the variation of temperature with time in the samples is to illustrate the influence of different laser treatments.

\section{The experiment}

\subsection{The samples and laser irradiation parameters}

$\mathrm{X}$-ray chemical analysis determined that there were 2.73 at.\% of Si, 1.02 at.\% of $\mathrm{Mn}, 0.3 \mathrm{at} . \%$ of $\mathrm{Cr}$, and 14 at.\% of carbon in the samples of grey cast iron before laser irradiation. X-ray diffraction and microscopic studies revealed that the iron was in the ferrite phase and that the carbon existed as graphite insertions.

The samples of grey cast iron for laser irradiation had an area of $50 \times 30 \mathrm{~mm}^{2}$ and a thickness of $10 \mathrm{~mm}$. For continuous scanned irradiation, a $\mathrm{CO}_{2}$ laser with a wavelength of $\lambda=10.6 \mu \mathrm{m}$ and $800 \mathrm{~W}$ of power was used. For pulsed irradiation, a $\mathrm{Nd}^{3+}$ YAlG laser with a wavelength of $\lambda=1.06 \mu \mathrm{m}$ and pulse energy of $8 \mathrm{~J}$ was used. Pulse duration was 1.5 and $4 \mathrm{~ms}$ and the period $100 \mathrm{~ms}$. For continuous action, changes in laser scan velocity allowed laser treatment times of 53, 110, and $875 \mathrm{~ms}$ to be obtained. For all cases, the diameter of the beam's spot on the sample's surface was $\leq 1-1.5 \mathrm{~mm}$.

The temperature of the surface and deeper layers during the laser irradiation was evaluated numerically by solving the heat conduction equation (Fig. 1a and b) [16]. Laser energy absorption by a sample's surface depends on the surface conditions. The absorption coefficient can vary within the limits of $0.2-0.4$. Therefore the energy to be absorbed by the surface was chosen so that the calculated temperature corresponded to the depth of the thermally affected or melted layer, as determined using an optical microscope, and a surface temperature of $T_{\mathrm{S}} \approx 2500-3000 \mathrm{~K}$, as measured with a pyrometer. The absorbed energy density used in the calculation was $\approx 1.8 \times 10^{8} \mathrm{~W} \mathrm{~m}^{-2}$ for the continuous action and $\approx 1.5 \times 10^{9} \mathrm{~W} \mathrm{~m}^{-2}$ and $\approx 5.6 \times 10^{8} \mathrm{~W} \mathrm{~m}^{-2}$, respectively, for the $1.5 \mathrm{~ms}$ and $4 \mathrm{~ms}$ duration pulsed laser irradiation.

All the temperature calculation data were made for pure iron. It would be difficult to take into account the graphite dissolution, carbon distribution, and 

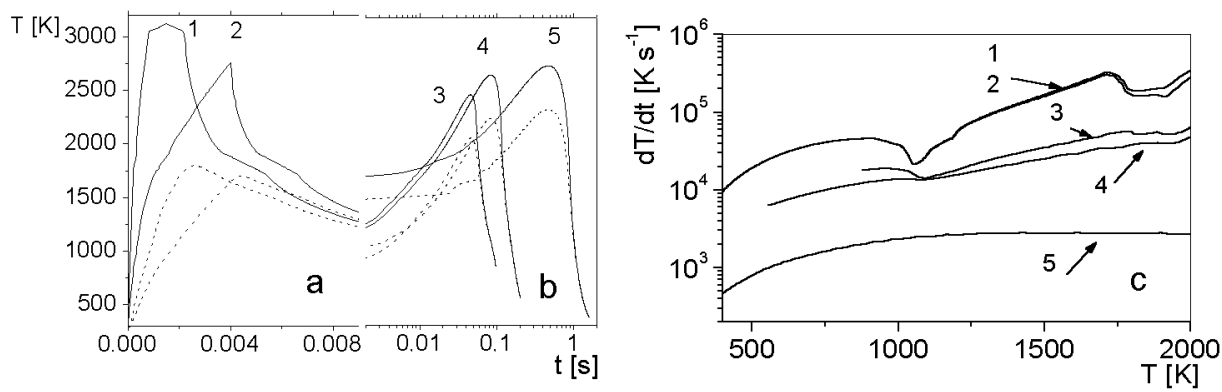

Fig. 1. The data of temperature modelling during laser irradiation of the samples: (a) pulsed, (b) continuous scanning irradiation, and (c) dependences of the quenching rate on temperature. In (a) and (b) solid line - the surface of the sample, dashed line - the depth of $0.1 \mathrm{~mm}$. Time of laser irradiation: $1-1.5 \mathrm{~ms} ; 2-4 \mathrm{~ms} ; 3-53 \mathrm{~ms}$; $4-110 \mathrm{~ms} ; 5-875 \mathrm{~ms}$.

carbide formation. On the other hand, because the carbide grains are small, their influence on thermal conductance is also small. For long irradiation times, when the amount of carbide is considerable, the quenching rate is limited by the velocity of the scan rather than by the properties of the material.

Although this temperature calculation is qualitative, it allows the influence of different laser irradiation to be compared. The quenching rate $\mathrm{d} T / \mathrm{d} t$ (Fig. 1c) was found to decrease with the increase in the laser irradiation time.

\subsection{X-ray diffraction studies}

The X-ray spectra, measured with a DRON-3 diffractometer at room temperature, are shown in Fig. 2. $\mathrm{Cu} K_{\alpha}$ radiation was used. The X-ray diffraction data were used for qualitatively evaluating the composition of the samples and the dimensions of the grains from different phases. The determination of the percentage of carbon $c_{c}^{\gamma}$ [at.\%] in the austenite was based on the change in the lattice constant [17]

$$
a_{\gamma}=0.3573+0.0007 c_{c}^{\gamma}
$$

where $a_{\gamma}$ is in nm. The influence of substitutional impurities of $\mathrm{Cr}, \mathrm{Mn}$, and Si on the lattice constant was substantially less than that of carbon and therefore it was ignored. The percentage of carbon in the martensite can be evaluated from the tetragonality of the martensite lattice $[17,18]$

$$
c / a=1+0.0096 c_{c}^{\alpha X}=1+0.0096 c_{c}^{\alpha} \eta,
$$

where $a$ and $c$ are the constants of the martensite's tetragonal lattice, $c_{c}^{\alpha}$ is the real percentage of carbon in the martensite, and $\eta$ is the parameter of the long-range order distribution of the carbon in the martensite. The percentage of carbon $c_{c}^{\alpha X}$ determined from Eq. (2) depends on the parameter $\eta$. The substitutional impurities 


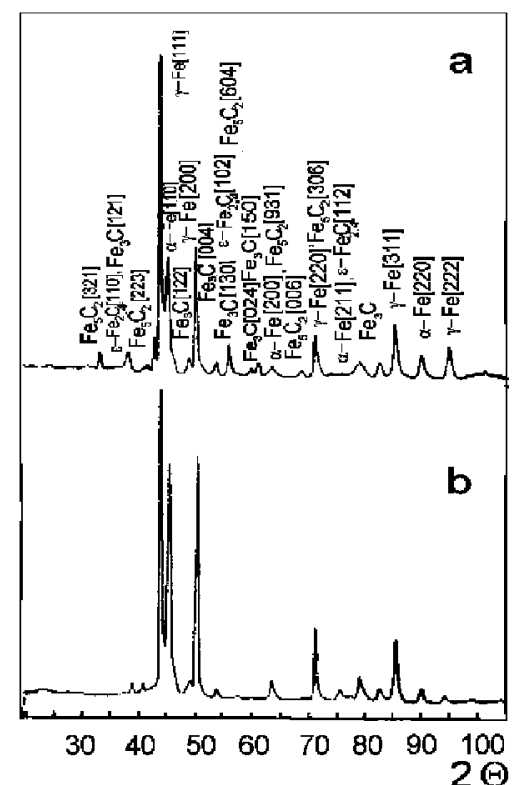

Fig. 2. The X-ray diffraction spectra: (a) surface, time of laser irradiation $-875 \mathrm{~ms}$; (b) surface, time of laser irradiation $-4 \mathrm{~ms}$.

(Cr, Mn, Si) affect $a$ and $c$ equally, therefore they do not impair the accuracy of the determination of the percentage of carbon in the $c / a$ ratio.

The dimension of the grains from different phases was evaluated using Debye-Scherrer equation [19]. The contributions of the grain size and internal strains to line broadening were distinguished on the basis of the ratio of the peak areas for the proper indices $(h k l)$ by the method presented in [19]. As regards the inaccuracy of the data associated with the possible presence of texture, we can only state that the grains were less than $50 \mathrm{~nm}$ in size.

In the X-ray diffraction spectra (Fig. 2), an apparent difference is observed for the continuous and pulsed laser irradiation of the material. The intensity and width of the peaks $\alpha$-Fe (110), (220), (211), (200) and $\gamma$-Fe (111), (200), (220), (311) depend on the laser irradiation time. A decrease or increase in the angle distance between the $\mathrm{X}$-ray lines indicates that large nonuniformities in the percentages of the impurities remained in the martensite and austenite after quenching. It is more difficult to determine the phase structure on the basis of the areas of the X-ray diffraction lines than on the basis of the Mössbauer spectroscopy data because of the need for long-range order for X-ray diffraction, the nonuniform distribution of the impurities, and the small dimensions of the grains.

\subsection{Mössbauer studies}

The Mössbauer spectra were measured with a constant acceleration spectrometer using a ${ }^{57} \mathrm{Co}$ source in the $\mathrm{Pd}$ matrix. The sample surface treated by 
laser irradiation was abraded and powder from the depth desired was used to prepare samples for Mössbauer studies. The samples were abraded in kerosene to prevent heating and possible changes in physical-chemical properties. The samples were collected from the surface and a depth of $0.1 \mathrm{~mm}$. The effective thickness of the Mössbauer samples was $20 \mathrm{mg} \mathrm{cm}^{-2}$ of iron. The spectra were measured at room temperature. The number of impulses for the spectrum point was $\approx 10^{6}$.

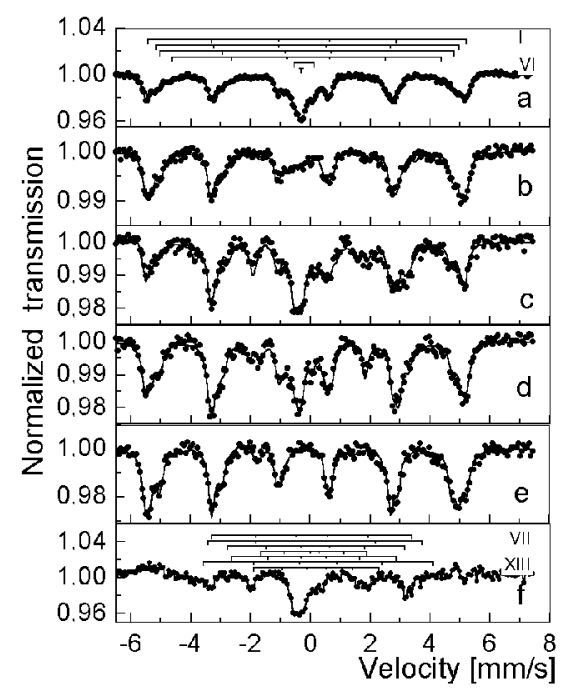

Fig. 3. Mössbauer spectra of the samples: (a) the surface, time of laser irradiation $1.5 \mathrm{~ms}$; (b) the depth $-0.1 \mathrm{~mm}$, time of laser irradiation $-1.5 \mathrm{~ms}$; (c) the surface, time of laser irradiation $-875 \mathrm{~ms}$; (d) the depth of $0.1 \mathrm{~mm}$, time of laser irradiation $-875 \mathrm{~ms}$ and (e) the primary sample, (f) weighted difference of the spectra of samples $\mathrm{c}$ and $\mathrm{e}$ (exclusion of martensite fraction). Subspectra: I-III - martensite; IV — iron-carbon clusters; V-VI - austenite, VII - carbide $\theta$-Fe ${ }_{3} \mathrm{C}$; VIII-X - carbide $\chi$-Fe $\mathrm{F}_{5}$, XI-XIII - carbide $\varepsilon-\mathrm{Fe}_{2.4} \mathrm{C}$.

The quantitative spectra analysis was carried out by fitting the experimental spectra (Fig. 3) to the subspectra of different phases. The determination of the iron phase structure was based on the areas of the subspectra (cf. Figs. 4-6). The parameters for the description of the Mössbauer spectra were taken from Refs. [5, 20]. For the description of austenite, the single peak and quadrupole doublet corresponding to the 0 and 1 carbon atoms in the first coordination sphere (I cs) were used. For martensite, three sextets were applied that included two iron atom coordination spheres with an $(0,0),(1,0),(0,1)$ arrangement of the substitutional and interstitial impurity atoms in the I and II cs (I-III subspectra in Table). Carbon atoms in I cs reduce the effective magnetic field on the nucleus of iron, $H_{\text {eff }}$, by $3 \mathrm{~T}$. Si, Cr, Mn impurity atoms reduce it by $\approx 2.7 \mathrm{~T}$ [21]. We assumed that the hyperfine splitting of the spectra was the same for all impurity atoms 
TABLE

Hyperfine fields of Mössbauer subspectra. The subspectra I-III correspond to martensite, IV to iron-carbon clusters, VII to cementite, VIII-X to $\chi$ - $\mathrm{Fe}_{5} \mathrm{C}_{2}$ and XI-XIII are subspectra of $\varepsilon$-Fe $\mathrm{Fe}_{2.4} \mathrm{C}$ carbide.

\begin{tabular}{c|c|c|c|c|c|c|c}
\hline \hline Subspectrum & I & II & III & IV & VII & VIII-X & XI-XIII \\
\hline & & & & & & 18.4 & 17.0 \\
$H_{\text {eff }}[\mathrm{T}]$ & \multirow{2}{*}{33.4} & 32.2 & 30.5 & $27-29$ & 20.8 & 22.2 & 23.7 \\
& & & & & & 11.0 & 13.0
\end{tabular}

in the I cs. Carbon and substitutional atoms in the II cs shift $H_{\text {eff }}$ by 1.2 T. Assuming that the distributions of both substitutional and interstitial impurities are independent, the probabilities of the surroundings of iron may be written as [13]

$$
\begin{aligned}
& P_{\mathrm{Fe}}(0,0)=P_{\mathrm{S}}^{\mathrm{M}}(0,0) \times P_{\mathrm{C}}^{\mathrm{M}}(0,0), \\
& P_{\mathrm{Fe}}(1,0)=P_{\mathrm{S}}^{\mathrm{M}}(1,0)+P_{\mathrm{C}}^{\mathrm{M}}(1,0), \\
& P_{\mathrm{Fe}}(0,1)=P_{\mathrm{S}}^{\mathrm{M}}(0,1)+P_{\mathrm{C}}^{\mathrm{M}}(0,1),
\end{aligned}
$$

where $P_{\mathrm{S}}^{\mathrm{M}}$ and $P_{\mathrm{C}}^{\mathrm{M}}$ are the probabilities for a substitutional impurity and for carbon atoms, respectively.

Substitutional and carbon atoms increase the effective magnetic field $H_{\text {eff }}(0,0)$ when there are no impurities in the two coordination spheres [21, 22]. The shifts induced by the substitutional impurities and the carbon are, respectively:

$$
\begin{aligned}
& \Delta H\left(0,0, c_{p}^{\alpha}\right) \approx 0.1 c_{p}^{\alpha} \\
& \Delta H\left(0,0, c_{c}^{\alpha}\right) \approx H_{\mathrm{eff}}(\mathrm{Fe}) \times 0.0039 c_{c}^{\alpha},
\end{aligned}
$$

where the magnetic field is expressed in Tesla $(\mathrm{T}), c_{p}^{\alpha}$ is the percentage of substitutional impurities, $c_{p}^{\alpha}$ is the carbon percentage in martensite, and $H_{\text {eff }}(\mathrm{Fe})$ is the value of the effective magnetic field for pure iron.

In the analysis of the Mössbauer spectra, those phases with a large percentage of carbon were described using sextets with the characteristic hyperfine magnetic fields. For the description of the cementite $\left(\mathrm{Fe}_{3} \mathrm{C}\right)$, the hyperfine magnetic field of the sextet was $20.8 \mathrm{~T}$. For other carbide $\varepsilon-\mathrm{Fe}_{2.4} \mathrm{C}, \chi-\mathrm{Fe}_{5} \mathrm{C}_{2}$ phases, the magnetic fields were within the intervals of $17-19 \mathrm{~T}, 22-23 \mathrm{~T}$, and 11-13 T [23-26] that corresponded to different characteristic sites (Table). The one more phase with a larger content of carbon and of iron-carbon clusters [27] was additionally included in the analysis of the Mössbauer spectra by the sextet with a hyperfine magnetic field within $26-28 \mathrm{~T}$.

\section{Results of the experiment and discussion}

\subsection{Austenite}

We studied the decay of high temperature $\gamma$-Fe-C solution (austenite) during quenching. The main point for the hardening of Fe-C systems is the saturation of $\gamma$-Fe with carbon and the creation of martensite. 
Retained austenite is a part of the high temperature $\gamma$-Fe solution that does not transform during quenching to martensite and carbides. This process depends on the rate of quenching. At a sufficiently high quenching rate, the concentration of carbon in high temperature austenite is reflected in retained austenite. A large amount of dissolved carbon stabilizes austenite, i.e., it prevents its transformation into martensite. The percentage of dissolved carbon (the solubility limit is 10.5 at.\% [28]) indicates whether the temperature is sufficiently high and whether irradiation time is sufficient to dissolve the graphite and mix the carbon with the iron.
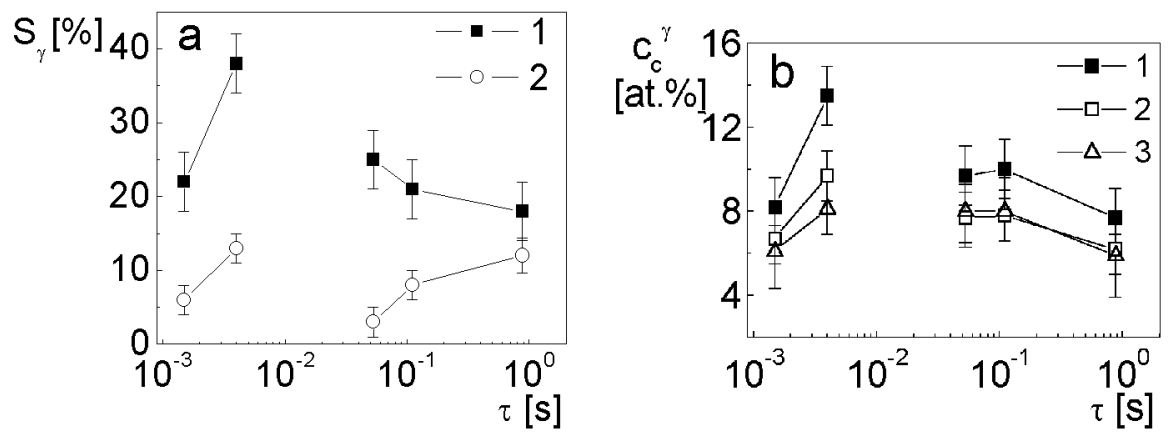

Fig. 4. The dependence of parameters of retained austenite on time: (a) the amount in the samples; 1 - the surface; 2 - the depth, $0.1 \mathrm{~mm}$; (b) percentage of carbon if carbon distribution is: 1 - random; 2 - repulsive; 3 - from X-ray data (from the change in the lattice constant).

The percentage of retained austenite depends on the laser irradiation time $\tau$ (Fig. 4). It is supposed that the amount of retained austenite in the zone affected by the laser irradiation is determined by two major processes, the saturation of the melt with carbon and the decay of austenite during quenching. In the case of continuous irradiation, the amount of retained austenite is limited mainly by the decay of austenite into different carbides. On the contrary, in the case of pulsed irradiation, the dissolution of the graphite and the saturation of the melt with carbon are the most important factors. Therefore, the percentage of retained austenite is less for the $1.5 \mathrm{~ms}$ pulse duration than for the $4 \mathrm{~ms}$ duration.

In the layer from a depth of $0.1 \mathrm{~mm}$, the temperature is lower compared to that of the surface (Fig. 1a and b). Therefore the saturation of the material with carbon is slower. In spite of this, for a laser irradiation time of $875 \mathrm{~ms}$, the amount of retained austenite on the surface and in the layer from a depth of $0.1 \mathrm{~mm}$ is nearly identical. It can be explained by the creation of a smaller amount of carbides in the deeper layer.

The carbon percentage, $c_{c}^{\gamma}$, in retained austenite may be evaluated according to the change in the lattice constant (1) and in two ways from the Mössbauer spectra. For the random distribution of carbon, the relative area of the singlet 
is $S_{\mathrm{A}}=P(0)=\left(1-c_{c}^{\gamma}\right)^{6}$, where $P(0)$ is the probability of iron atoms that have no carbon atoms in the I cs. For the distribution of the repulsive type, it is $S_{\mathrm{A}}=P(0)=1-6 c_{c}^{\gamma}[28]$.

In the case of random carbon distribution, the probability, corresponding to the solubility limit, of finding no carbon atoms in the $\mathrm{I}$ cs is $P(0)=0.51$. The experimental $P(0)$ values are within $0.42-0.63$, which means that the carbon percentage can exceed the solubility limit. In Refs. [28, 29], a repulsive type of carbon distribution was confirmed by means of Mössbauer spectroscopy. When quenching rates are high, the random distribution can probably be frozen. The values of $c_{c}^{\gamma}$ found by different methods are indicated in Fig. 4b. The best coincidence is observed between the $c_{c}^{\gamma}$ values based on the $\mathrm{X}$-ray diffraction data for the changes in the lattice constant (Eq. (1)) and the repulsive model for the carbon distribution. It has been seen that if the percentage $c_{c}^{\gamma}$ is above 5 at.\%, an $\mathrm{Fe}_{8} \mathrm{C}$ type structure is formed [28]. For the continuous scanned irradiation, the percentage of carbon in austenite tended to decrease with the increase in the irradiation time.

\subsection{Martensite}

The mechanical, magnetic, and electric macroscopic properties of the alloy mainly depend on the structure of martensite, or rather on a conglomerate of martensite and carbides. The variation in the amount of martensite in the alloy with the time is shown in Fig. 5. The parameter of the martensite lattice according to the X-ray diffraction data is close to $0.286 \mathrm{~nm}$ and the tetragonality factor $c / a$ is about $1.007-1.018$. This indicates that the martensite lattice in the samples is close to cubic. The percentage $c_{c}^{\alpha X}$ according to Eq. (2) is 1-2 at.\% . It is the real carbon percentage if the factor of the long-range order is $\eta=1$. The long-range order can decrease as martensite gets older due to the diffusion of

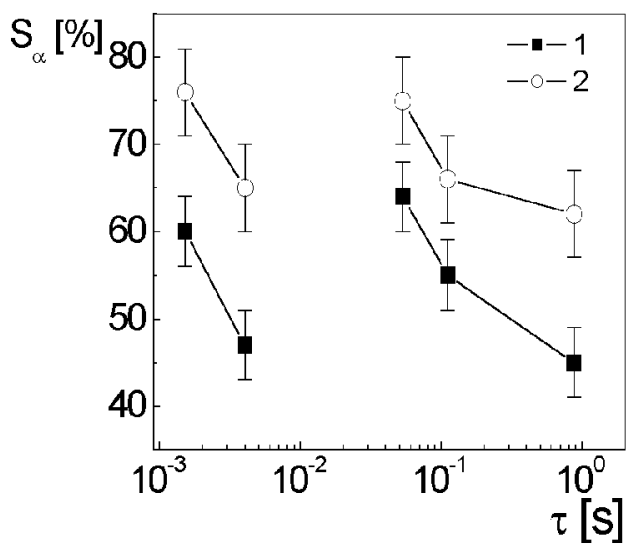

Fig. 5. The dependence of the amount of martensite on time: 1 - the surface, 2 the depth, $0.1 \mathrm{~mm}$. 
carbon atoms between different sublattices. The tetragonality can also decrease due to annealing effects when scanning the neighbouring area with a laser. The carbon percentage from the Mössbauer data $c_{c}^{\alpha M}$ and substitutional impurities percentage $c_{p}^{\alpha}$ can also be evaluated on the basis of Eqs. (3a, b) using the least square method. The values $c_{c}^{\alpha M}$ and $c_{p}^{\alpha}$ are found at the minimum of the sum of the squares of the difference between the experimental and theoretical probabilities $P_{\mathrm{Fe}}(0,0)$ and $P_{\mathrm{Fe}}(1,0)$. Because the magnetic field shifts induced by $\mathrm{Si}, \mathrm{Cr}$, and $\mathrm{Mn}$ are similar, only the general percentage of substitutional impurities in martensite can be found. The percentage of substitutional impurities $c_{p}^{\alpha}$ evaluated on the basis of X-ray chemical analysis is 4.1 at.\%. After laser irradiation, $c_{p}^{\alpha}$ found from Mössbauer data is 2.9-3.8 at.\%. Samples affected by laser irradiation have a rather large percentage of carbide in the alloy. Cr and Mn, which have a greater affinity for carbon than iron, can move from martensite to carbides. Moreover, Si stabilizes $\varepsilon$ carbide, and therefore the percentage of Si can decrease in martensite.

The carbon percentage, evaluated on the basis of the Mössbauer data, $c_{c}^{\alpha M}$ is 2.7-3.2 at.\%. An independent evaluation of $c_{c}^{\alpha M}$ and $c_{c}^{\alpha X}$ enables the long-range order parameter $\eta=c_{c}^{\alpha X} / c_{c}^{\alpha M}=0.2-0.6$ to be found. What are the reasons for the discrepancy in $c_{c}^{\alpha X}$ and $c_{c}^{\alpha M}$ ? In addition to the aging and annealing effects in martensite, thermodynamic peculiarities of the carbon distribution exist. Atoms of carbon in martensite may occupy one of three possible octahedral interstices [18]. The reason for the tetragonality of the lattice is that carbon atoms are mostly in the same octahedral sublattice. When only one sublattice is occupied, the distribution can be considered fully ordered $(\eta=1)$. This distribution occurs when intergranular stress energy is minimal. Inner thermodynamic equilibrium is not achieved due to the two empty sublattices but can be reached when carbon is distributed among all the sublattices. A minimum of intergranular energy is achieved when the volume is filled with disperse phases of small-dimensioned iron-carbon clusters or carbides. If carbon is randomly distributed between the interstices, the martensite has a cubic symmetry. This state is observed after the alloying carbon with the $\alpha-\mathrm{Fe}[5]$.

\subsection{Carbides}

The results of $\mathrm{X}$-ray diffraction (Fig. 2) indicate that some carbon-rich phases of $\varepsilon-\mathrm{Fe}_{2.4} \mathrm{C}, \chi-\mathrm{Fe}_{5} \mathrm{C}_{2}$, and $\theta-\mathrm{Fe}_{3} \mathrm{C}$ carbides [23-26] do exist. On the other hand, the remaining ferromagnetic part of the Mössbauer spectra, after excluding the martensite (Fig. 3f), may be attributed mainly to cementite $\theta-\mathrm{Fe}_{3} \mathrm{C}$ but, since this part of the Mössbauer spectra is broad and the different carbide subspectra lines (Table) overlap, it does not contradict the existence of the $\varepsilon-\mathrm{Fe}_{2.4} \mathrm{C}$ and $\chi-\mathrm{Fe}_{5} \mathrm{C}_{2}$ carbides.

In the samples, carbides exist in the form of the disperse inclusions. Since the crystalline structure is complicated, the long-range order can be easily broken. Therefore a long irradiation time is needed for the creation of the ordered large 
grain carbide structure. The X-ray diffraction studies confirm the presumption of a dispersed structure. For a short irradiation time, the peaks in the X-ray spectra are broadened and their intensities are lower. The major carbide phase, cementite $\left(\theta-\mathrm{Fe}_{3} \mathrm{C}\right)$, is explicitly seen in the X-ray diffraction as well as in the Mössbauer spectra. Attention should be paid to the temperature conditions during the creation of the carbide phases in the samples. The high-temperature $\theta-\mathrm{Fe}_{3} \mathrm{C}$ phase is formed mainly in the melt and the other carbide phases can form only during quenching at lower temperatures (Fig. 1c). The $\varepsilon-\mathrm{Fe}_{2.4} \mathrm{C}$ carbides are unstable but can decay efficiently at above $600 \mathrm{~K}[30]$ to $\chi-\mathrm{Fe}_{5} \mathrm{C}_{2}$ carbide due to tempering when the laser beam scans parallelly through the neighbouring area. The peaks of $\chi$ - $\mathrm{Fe}_{5} \mathrm{C}_{2}$ are clearly seen in the $\mathrm{X}$-ray spectra for the samples affected by long irradiation time (Fig. 2a).

The Mössbauer subspectra for $\varepsilon$ and $\chi$ carbides (Table) are hardly distinguishable due to their low intensity and close average hyperfine magnetic fields. For $\varepsilon$ carbide, the average hyperfine magnetic field is $\bar{H}=18 \mathrm{~T}$ and, for $\chi$ carbide, $\bar{H}=18.5 \mathrm{~T}$. The average hyperfine magnetic fields are used for distinguishing $\varepsilon$ and $\chi$ carbides from cementite and measuring their amounts. The percentage of $\varepsilon$ and $\chi$ carbides increases as the laser irradiation time increases and the melt after crystallisation remains longer within the temperature range for the formation of $\varepsilon$ and $\chi$ carbides (Fig. 6). For $\tau \leq 53 \mathrm{~ms}$, the percentage of all, $\varepsilon, \chi$, and $\theta$, carbides is within the limits of $10-20 \%$ and for $\tau>53 \mathrm{~ms}$, their percentage increases up to $35 \%$ (Fig. 6).

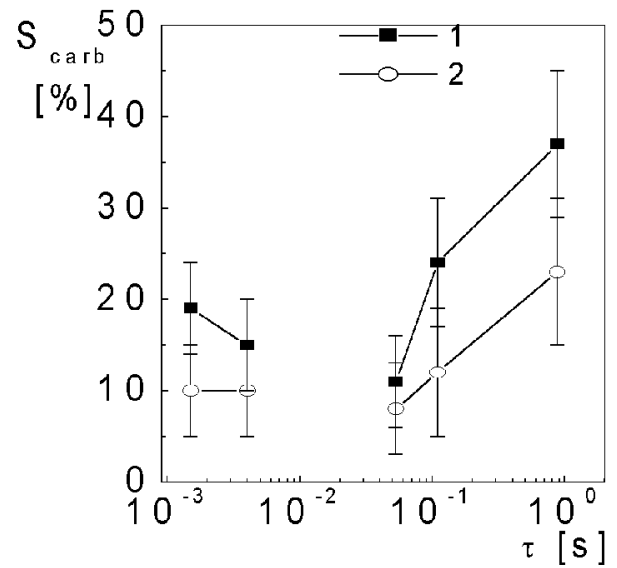

Fig. 6. The dependence of the amount of carbides in the surface on time: 1 - the amount of all carbides; 2 - the amount of $\varepsilon$ and $\chi$ carbides.

The other carbon-rich phase that corresponds to the $H_{\mathrm{eff}}=27-28 \mathrm{~T}$ subspectrum is iron-carbon clusters or carbon multiplets [27]. In iron-carbon clusters, iron atoms, which are in a disordered $\alpha$-Fe lattice, have two carbon atoms in 
the I cs. As the insertion of carbon atoms creates tensions, more favourable positions for iron-carbon clusters are defects such as dislocations. A probable place for them is the boundary regions between the grains [13]. The percentage of clusters was $3-7 \%$.

\section{Conclusions}

The application of different laser treatment types and times allowed the phase composition in the surface of grey cast iron to be altered. An increase in the laser irradiation time caused an increase in the amounts of the phases with a larger percentage of carbon. The calculation of the carbon content in the phases showed that, for the largest time of $875 \mathrm{~ms}$, almost all the graphite was dissolved in the surface and was found in the iron phases.

In Ref. [13] additional application of ultrasound treatment during laser irradiation showed that ultrasound influences carbon distribution and stimulates austenite decay and the transformation to iron-carbon clusters. In this study, an increase in the time of the applied laser treatment caused the decay of the austenite and the creation of $\varepsilon$ and $\chi$ carbides. This can be explained by the increase in the quenching time. The results of temperature modelling allowed the quenching rate of $10^{3}-10^{5} \mathrm{~K} \mathrm{~s}^{-} 1$, which depended on the scanning velocity of the laser beam, to be evaluated.

Mössbauer spectroscopy and X-ray diffraction allowed the phase composition to be identified and enabled the percentage and the type of impurity distribution in the phases to be evaluated. The decrease in the tetragonality of the martensite from the value that would correspond to the perfect long-range order $(\eta=1)$ was observed for all the laser irradiation times. The best coincidence of Mössbauer spectroscopy and $\mathrm{X}$-ray diffraction results provided evidence that the carbon distribution in the austenite was repulsive. The martensite had 2.7-3.2 at.\% of carbon and the austenite $6-10$ at.\% of carbon.

\section{References}

[1] R. Valiev, V. Bochkov, Sh. Bashkirov, E. Romanov, V. Chistjakov, Hyperfine Interact. 69, 589 (1991).

[2] O. Schneeweis, K. Volenik, Hyperfine Interact. 46, 527 (1989).

[3] Kou Sindo, K.D. Sun, Y.P. Le, Metall. Trans. A 14, 643 (1983).

[4] A. Amulevicius, M. Balciuniene, B. Petretis, D. Juzakenas, Metalloved. Term. Obrab. Met. 1, 12 (1994).

[5] A. Amulevicius, M. Balciuniene, S. Grigaliunas, B. Petretis, Fiz. Met. Metalloved. 76, 94 (1993).

[6] D.M. Gureyev, N.G. Kakovkina, S.I. Mednikov, E.S. Romanov, J. Sov. Laser Res. 12, 352 (1991). 
[7] Y. Isshiki, J. Shi, H. Nakai, M. Hashimoto, Appl. Phys. A 70, 395 (2000).

[8] A.B. Lisenko, N.N. Kozina, I.S. Miroshnichenko, G.V. Borisova, Metalloved. Term. Obrab. Met. 12, 10 (1995).

[9] A. Amulevicius, M. Balciuniene, B. Petretis, R. Pileckis, Thin Solid Films 229, 192 (1993).

[10] A. Amulevicius, M. Balciuniene, B. Petretis, R. Pileckis, Thin Solid Films 240, $60(1994)$

[11] L. Lanotte, V. Iannotti, J. Appl. Phys. 78, 3531 (1995).

[12] V.N. Gridnev, V.G. Gavriliuk, V.M. Nadutov, Fiz. Met. Metalloved. 60, 590 (1985).

[13] A. Amulevicius, K. Mazeika, A. Daugvila, J. Phys. D, Appl. Phys. 33, 1985 (2000).

[14] J. Desimoni, Hyperfine Interact. 134, 93 (2001).

[15] A. Amulevicius, R. Davidonis, R. Kanapenas, J. Reksnis, J. Tamoliunas, E. Jakstas, Lithuan. J. Phys. 29, 495 (1989).

[16] A.G. Grigoryanets, Principles of Laser Processing of Materials, Mashinostroenie, Moskow 1989, p. 304 (in Russian).

[17] L.I. Mirkin, X-ray Structure Estimation of Materials for Mechanism Building, Handbook, Mashinostroenie, Moskow 1979, p. 134 (in Russian).

[18] A.G. Hachaturyan, Theory of Phase Transitions and Structure of Solid Solutions Nauka, Moskow 1974, p. 384 (in Russian).

[19] L.I. Mirkin, X-ray Analysis of Polycrystal Structure, Handbook, Fizmatgiz, Moskow 1961, p. 863 (in Russian).

[20] V.V. Chekin, Mössbauer Spectroscopy of Iron, Gold and Tin, Energija, Moscow 1981, p. 107 (in Russian).

[21] S.M. Dubiel, W. Zinn, J. Magn. Magn. Mater. 45, 298 (1984).

[22] J. Lauermannova, in: Proc. 5-th Inter. Conf. on Mössbauer Spectroscopy, Part. 2, Eds. M. Hucl, T. Zemcik, Nuclear Information Centre, Praha 1975, p. 302.

[23] S.M. Kanetkar, P.P. Path, S.V. Ghaisas, S.B. Ogale, Hyperfine Interact. 41, 587 (1988).

[24] P.S. Arenc, Yu.V. Maksimov, I.P. Suzdalev, in: Proc. 5-th Inter. Conf. on Mössbauer Spectroscopy, Part. 2, Eds. M. Hucl, T. Zemcik, Nuclear Information Centre, Praha 1975, p. 328.

[25] E.J. Miola, S.D. Souza, M. Olzon-Dionysio, D. Spinelli, M.R.F. Soares. M.A.Z. Vasconcelles, C.L. dos Santos, Mater. Sci. Eng. A 256, 60 (1998).

[26] G. Le Caer, E. Bauer-Grosse, Hyperfine Interact. 47, 55 (1989).

[27] J.M.R. Genin, O.N.C. Uwakweh, J. Schanen, Ph. Bauer, Hyperfine Interact. 41, 575 (1988).

[28] Ph. Bauer, O.N.C. Uwakweh, J.M.R. Genin, Hyperfine Interact. 41, 555 (1988).

[29] A.N. Timoshevskii, V.A. Timoshevskii, B.Z. Yanchitsky, J. Phys. Condens. Matter 13, 1051 (2001).

[30] J.M.R. Genin, G. Le Caer, A. Simon, in: Proc. 5-th Inter. Conf. on Mössbauer Spectroscopy, Part. 2, Eds. M. Hucl, T. Zemcik, Nuclear Information Centre, Praha 1975, p. 318. 